$\underline{\text { Review }}$

\title{
Severe acute respiratory syndrome coronavirus 2 vaccines - a review of adverse effects and safety profiles
}

\author{
VW Premaratne, R Farook, SD Dharmaratne \\ Sri Lankan Journal of Infectious Diseases 2022 Vol.12(1):E2 1-11 \\ DOI: http://dx.doi.org/10.4038/sljid.v12i1.8413
}

\begin{abstract}
Since the emergence of SARS CoV-2 in 2019, the global population has had to implement major lifestyle changes in order to circumvent death and severe morbidity caused in the wake of the virus, as well as endure the consequences on the economy and social wellbeing. However, restrictions posed by the pandemic have not deterred scientists from engineering a solution to this in the form of vaccines against SARS CoV-2. Although their efficacy has been proven, recent reports have claimed that these vaccines may not be as safe as they are promoted to be, and that they may cause adverse effects that outweigh their benefits. This article offers an objective perspective of the unfavourable side effects of five widely used vaccine candidates for SARS CoV-2, BNT162b2, mRNA 1273, ChAdOx1 nCoV-19, Sputnik V and BBIBP CorV, to highlight that the panic associated with vaccination is unjustified in light of recent developments.
\end{abstract}

Keywords: Covid 19, Vaccination, Safety

\section{Introduction}

\section{Emergence and Impact of SARS CoV-2}

Since the WHO issued a warning on the probability of a global health crisis due to the and extensive spread of a new virus strain worldwide, the novel coronavirus, scientifically named Severe Acute Respiratory Syndrome Coronavirus 2 (SARS CoV-2), has gained public attention. ${ }^{1}$ In the past two decades, SARS CoV-1 and Middle East Respiratory Syndrome (MERS) have also given rise to pandemics, and they share their lineage with SARS CoV-2. All three are beta coronaviruses. However, SARS CoV-1 and MERS had far less of an outreach, affecting a few geographically related countries and resulting in only 1632 deaths combined. ${ }^{2}$ In comparison, the

${ }^{1}$ Faculty of Medicine, University of Peradeniya, Sri Lanka

Address for correspondence: Dr. V.W. Premaratne, Faculty of Medicine, University of Peradeniya, Sri Lanka, Telephone: +0094713465011 E-mail: Wihangapremaratne@gmail.com

https://orcid.org/0000-0003-3405-9775

Received 2 August 2021 and revised version accepted 27 October 2021. Published 7 February 2022

C) (7)

use, distribution, and reproduction in any medium, provided the original author and source are credited. 
current death toll due to SARS CoV-2 has increased up to 4.9 million worldwide, as of October 2021, and is still increasing steadily. ${ }^{3}$

SARS CoV-2 can be transmitted by direct, indirect or close contact. The incubation period is known to be 2-14 days. ${ }^{4}$ It is thought that the pathogenic mechanism by which SARS CoV-2 causes target organ damage is by modulation of the activity of the angiotensin-converting enzyme 2 (ACE 2) receptor and enabling invasion. ACE 2 receptors are abundantly found on tissues of the lungs, gastrointestinal tract, vessels, heart and kidneys, resulting in a variety of symptoms such as fever, cough, shortness of breath, muscle or body aches, headache, anosmia, sore throat, vomiting, and diarrhea. $^{2,4-7}$

Apart from direct damage by the virus, the exaggerated immune response to viral antigens releases chemical mediators of inflammation, causing immune mediated damage leading to the cytokine storm syndrome. ${ }^{4}$ This results in acute respiratory distress syndrome and respiratory failure, which leads to death in a genetically predisposed group of patients with severe disease. These immune responses can also lead to vasculitis, thrombosis, cerebrovascular disease, multi-organ dysfunction and shock. ${ }^{4,8}$

SARS CoV-2 nucleic acid detection by the polymerase chain reaction (PCR) remains the gold standard for diagnosis. ${ }^{4-9-11}$ Immunological detection methods can also be used but their sensitivity and specificity have been found to be low. ${ }^{12}$ Treatment is mainly supportive but some therapeutic options include the use of antivirals such as ribavirin, use of convalescent plasma and certain monoclonal antibodies, of which studies are still underway. ${ }^{13}$

As an initiative towards reducing the burden of morbidity and mortality associated with SARS CoV-2, scientists developed vaccines to promote primary prevention of the disease, and their efforts were successful in producing vaccines with high efficacy. However there has been recent speculation whether these vaccines lead to more debilitating consequences than the virus itself. This review aims to compare five widely used vaccines that are currently available, on their reactogenicity and safety profiles.

\section{Vaccines and their uncharted territories}

Currently the only fully licensed vaccine for SARS CoV-2 is Pfizer ${ }^{2}$, although 127 vaccines are in clinical development. ${ }^{14}$ Several vaccine candidates are in stage 4 clinical trials, of which some have been approved in different countries for emergency use. ${ }^{14,15}$ The precise reason that full approval has not been given for some vaccines is that the usual process of years of clinical research and multinational controlled trials has been expedited and cut short by the urgent need of a remedy. Vaccination has therefore been carried out worldwide despite their unexplored outcomes. According to the Our World in Data Statistics database on SARS CoV-2, the vaccine candidates that are commonly administered are Comirnaty/BNT162b2 (Pfizer), Vaxzevria/AZD1222/ Covishield/ChAdOx1 nCoV-19 (AstraZeneca), mRNA 1273 (Moderna), BBIBP-CorV (Sinopharm) and Sputnik V (Gamaleya). ${ }^{16}$

BNT162b2, manufactured by the United States of America (USA) based enterprise Pfizer and the German company BioNTech, as well as the candidate vaccine mRNA 1273 created by the USA 
based company Moderna, have compositions which are very alike. They are both gene-based vaccines with lipid nanoparticle-formulated, nucleoside-modified mRNA which encode the membrane-anchored SARS CoV-2 full-length spike protein which is stabilized by conformational changes. ${ }^{17,18}$ This alteration induces much higher neutralizing antibody titers in terms of quality and quantity. ${ }^{19}$ The candidate vaccines ChAdOx $1 \mathrm{nCoV}-19$ and Sputnik V are similar in that they use adenoviral vectors to deliver the full length SARS CoV-2 spike protein gene, but without any conformational changes. ${ }^{20,21} \mathrm{ChAdOx} 1 \mathrm{nCoV}-19$, designed by the University of Oxford, England, and mass produced by the British-Swedish Company AstraZeneca, employs a replication-deficient chimpanzee adenoviral vector (ChAdOx1) for carriage ${ }^{22,23}$ while Sputnik V, created by the Gamaleya research institute, Russia, uses a heterologous recombinant adenovirus approach by means of two different adenoviral serotypes, Ad26 and Ad5, for the expression of the same target vector. ${ }^{20}$ The candidate vaccine BBIBP CorV is an inactivated vaccine manufactured by Sinopharm, based in China. Inactivated vaccines are created by growing SARS CoV-2 in cell culture, followed by chemical inactivation. ${ }^{24}$

\section{Safety of vaccines - what we know so far}

SARS CoV-2 vaccines have not been in use for an adequate duration of time to assess their comprehensive safety profiles. However, potential safety risks have been predicted taking into consideration the similarity of their compositions and generated immune responses to outcomes of previous vaccines. There are theoretical concerns that the disease can be heightened due to antibody-dependent enhancement, where the binding antibodies produced in response to the SARS CoV-2 virus forms antibody-virus complexes which augment viral entry into immune cells. This has led to an opinion that a type $1 \mathrm{~T}$-helper (Th1)-based immune response is favourable in terms of safety of candidate vaccines. ${ }^{2,22,23}$ Conversely, it is thought that Th2 responses are associated with non-neutralizing antibody responses and poor outcome. However, to date, enhanced disease has not been observed in preclinical models or humans with any vaccines currently in development. $^{2}$

Safety of candidate vaccines can also be assessed by their side effect profile in terms of local reactogenicity and systemic reactogenicity. Certain fatal adverse effects have been linked to some vaccines which have caused concerns. However, any added risk compared to the population risk has yet to be proved.

T cell responses

In-depth studies assessing the $\mathrm{T}$ cell responses of individual vaccines are far and few. It has been found that all vaccine candidates showed favourable Th1-skewed immune responses albeit with some contradictory evidence in some studies assessing the Sinopharm vaccine. ${ }^{15,18,25}$ The T cell responses induced by the $\mathrm{ChAdOx} 1 \mathrm{nCoV}-19$ vaccine peaked at day 14 but were found to be lower in those who were more than 70 years. ${ }^{23,26}$ The Th2 responses with the vaccine were minimal regardless of age or dose. ${ }^{15}$

In a study relating to the $\mathrm{T}$ cell responses generated by the vaccine candidate Sinopharm, the authors had detected no $\mathrm{T}$ cell activity with the vaccine. However specific results were not available. ${ }^{26}$ In more recent studies, it was found that when comparing Sinopharm and Pfizer 
vaccines, the cumulative $\mathrm{T}$ cell response was more in the Pfizer injected individuals than in Sinopharm vaccinated individuals. However, the Sinopharm vaccine was able to elicit a wider range of $\mathrm{T}$ cell responses to SARS CoV-2 structural proteins which was comparable to convalescent serum, while the Pfizer vaccine only developed a response to the spike protein. Therefore the authors concluded that both vaccines were capable of eliciting $\mathrm{T}$ cell responses but the Pfizer vaccine elicited a stronger but narrower response. ${ }^{27}$ These findings of $\mathrm{T}$ cell responses by the Sinopharm vaccine were further confirmed in another study. ${ }^{28}$

In a study assessing $\mathrm{T}$ cell responses in the two mRNA vaccines (Pfizer and Moderna), it was found that both the vaccines showed broad $\mathrm{T}$ cell responses to the SARS CoV-2 spike protein which were able to effectively recognize variants of the SARS CoV-2 virus as well as the ancestral virus, providing cross-protection. ${ }^{29}$

The vaccine candidate Sputnik V showed increased $\mathrm{T}$ cell responses in all vaccine recipients, and this finding was consistent with the long term persistence of the generated immune response. ${ }^{20,30}$

Local Reactogenicity

The local adverse effects produced by the AstraZeneca vaccine were injection-site pain, tenderness, warmth, redness, swelling, induration and itch. Injection-site pain and tenderness were the most common solicited local adverse reactions and occurred most frequently in the first 48 hours following vaccination. At least one local adverse effect was reported after the first standard dose of AstraZeneca by $88 \%$ of participants in the $18-55$ years group, $73 \%$ of participants in the 56-69 years group and $61 \%$ of participants in the 70 years and older group. Similar statistics were reported after the boost vaccination with values of $76 \%, 72 \%$ and $55 \%$ in the respective groups. None of these symptoms were considered to be severe by the research team. ${ }^{22,23}$ This shows that fewer adverse events were detected in older adults and following the booster vaccine. These side effects were also found to be less with a lower dose, but this may be due to the fact that the injection volumes also differed. However, the local effects were considerably higher compared to the control group.

In clinical trials done to assess the Pfizer vaccine, overall, the recipients reported more local reactions than the placebo controls. The adverse effects included injection site pain, redness and swelling, of which injection site pain was the most commonly reported reaction. Pain was reported by $83 \%$ of younger individuals and $71 \%$ of those over 55 years old, respectively, which decreased to $78 \%$ and $66 \%$ after the second dose. The authors concluded that the majority of the local reactions were mild to moderate in severity and resolved within a short period of time. ${ }^{17}$ These findings are comparable to those of the AstraZeneca vaccine.

Moderna published their safety data on local reactions, stating that adverse effects were present in more than $50 \%$ of participants and the commonest reaction was pain at the injection site. Even though these effects were mild, they reported that they were dose dependent and commoner after the second dose, in contrast to the AstraZeneca and Pfizer vaccines. ${ }^{18}$

Clinical trials assessing the Sputnik V vaccine reported that there were no serious adverse effects recorded ${ }^{20}$ and that injection site reactions were the commonest reported adverse event. ${ }^{21}$ 
Studies of the Sinopharm vaccine reported that the safety profile was excellent with no grade 3 reactions. ${ }^{31}$ Another study reported that the most common local effect reported was injection site pain which was present in $24 \%$ of participants between the ages of 18 and 59 years given the vaccine, and only $6 \%$ in placebo controls. Other reactions included injection site swelling and itch. The study further noted that the effects were higher with increased dose and lower with increasing age as shown with the other vaccines. Another fact that the study highlighted was that induration at the injection site was seen mainly in older adults compared to younger participants. ${ }^{24}$

\section{Systemic Reactogenicity}

Systemic reactogenicity has been reported extensively for each vaccine. The AstraZeneca vaccine has reported an acceptable safety profile. ${ }^{22}$ However systemic reactions were reported to be commoner in the vaccine arm of participants and further described to be $86 \%$ in the 18-55 years age group, $77 \%$ in the 56-69 years age group and $65 \%$ in the 70 years and older age group. This demonstrates the same trend seen with local reactions where there were reduced adverse effects with age. ${ }^{22,23}$ The documented systemic adverse effects were fever, muscle ache, malaise, joint pain, fatigue, nausea, chills and headache, with fatigue, headache, fever and myalgia topping the list ${ }^{23,31}$. The effects reduced with a boost dose as seen with the local reactions. ${ }^{23}$ Across all age groups the adverse effects were dose-dependent. ${ }^{23}$ In a study comparing the AstraZeneca vaccine and a licensed meningitis vaccine (MenACWY), the authors concluded that the AstraZeneca vaccine had a poorer safety profile than the meningitis vaccine, independent of any treatment given to alleviate systemic symptoms. ${ }^{31}$ In a study based in the United Kingdom, the reported rate of anaphylaxis for the vaccine was found to be 23 per million doses. The same study compared this rate with that of Pfizer and there was no significant difference of reported cases ( 24 per million doses). ${ }^{32}$

Pfizer also reported that systemic events were recounted more often by younger vaccine recipients than older participants. However, in contrast to the findings on local effects, the systemic adverse reactions were commoner after the second dose compared to the first. The most commonly reported effects were fatigue and headache, which was reported by $59 \%$ and $52 \%$ of participants respectively. Other effects included fever and lymphadenopathy. Severe systemic events were reported in less than $2 \%$ of vaccine recipients and the investigators had established that they were not considered to be due to the vaccine. ${ }^{17}$ In another study, the commonest adverse effect was reported to be fever which was seen in more than $70 \%$ of individuals. There was an increase in symptoms with increased dose and a booster dose, confirming the findings of the previous study. ${ }^{31}$ Allergic reactions to the Pfizer vaccine have also been assessed in cohorts of patients. One study showed that among 6146 subjects, 118 were identified as having a history of severe allergy, of whom $14 \%$ compared with $0.2 \%$ of normal individuals developed hypersensitivity reactions following vaccination. Skin manifestations were the commonest. Only one person, belonging to the normal group, developed grade-4 anaphylaxis. ${ }^{33}$ In another study, among a group of 429 individuals who were defined as highly allergic, only 6 individuals (1.4\%) developed minor allergic reactions and 3 individuals $(0.7 \%)$ developed anaphylaxis. ${ }^{34}$ Authors of both studies concluded that the Pfizer vaccine can be relatively safely administered in people, even those with a history of severe allergic reactions. 
Studies on the Moderna vaccine have reported adverse events in more than half the participants which included fatigue, chills, headache and myalgia. Systemic adverse events were more common after a second dose and with a higher dose. ${ }^{18,31}$ Severe solicited reactions, when reported, were labeled unrelated to the vaccine. One unsolicited reaction that was related to the vaccine was reported to be decreased appetite ${ }^{15}$. Another study reported that systemic events were reported in $100 \%$ of participants after the boost dose of vaccine with the standard dose. ${ }^{31}$ In one adverse events database it was found that of 4 million people vaccinated with the Moderna vaccine, only 1266 $(0.03 \%)$ adverse events were reported, of which $10(\%)$ were due to anaphylaxis. ${ }^{35}$

Studies on the Sputnik V vaccine have reported serious adverse events such as renal colic, deep vein thrombosis and extremity abscess, but these were considered to be unrelated to the vaccine, further evidenced by the fact that a higher percentage of the placebo participants had effects compared to the vaccine group ${ }^{20}$. The commonest adverse events were a flu-like illness, headache and malaise. Two deaths in the vaccination arm were reported due to SARS CoV-2 infection but the patients were thought to be already infected prior to enrollment in the study despite an initial negative PCR test. ${ }^{21}$

The Sinopharm vaccine trials reported that at least one systemic adverse reaction was reported by $29 \%$ of vaccine recipients. The commonest reaction was fever, among others such as fatigue, loss of appetite, nausea, constipation, headache, vomiting and mucocutaneous abnormalities. Comparable findings to the other vaccines were present, with increase of adverse effects with higher doses and lower effects with increasing age. ${ }^{24}$ In both Sputnik V and Sinopharm vaccines, no vaccine-specific data on allergic reactions and post vaccination anaphylaxis has been published through large scale clinical trials or databases. ${ }^{32}$

Apart from the solicited, seemingly mild systemic side effects of the vaccines, another complication that caused public unrest was the thromboembolism risk due to certain vaccine candidates. The AstraZeneca vaccine had been temporarily withheld in some countries due to this. In a study done in Denmark and Norway, 281,264 people who received the AstraZeneca vaccine were assessed for the standardized morbidity ratios for arterial and venous thromboembolic events, including cerebral venous thrombosis, as well as thrombocytopaenia and coagulation disorders. There were 59 venous thromboembolic events reported in the vaccine arm compared with the 30 that were expected through estimated incidence rates in the population, but only 15 deaths were observed in the vaccine cohort compared with 44 expected. The study concluded that the rates of venous thromboembolic events, including cerebral venous thrombosis, were significantly higher than expected. However the other parameters including thrombocytopenia/coagulation disorders and bleeding showed only slight increments of absolute risk, probably due to increased surveillance of vaccine recipients. ${ }^{36}$ In another retrospective study done by analyzing spontaneous reports submitted to an adverse events database, there were 54,571 reactions reported, of which 28 were associated with thrombotic complications. Three fatalities were due to pulmonary embolism and one due to thrombosis. Compared to the number of people who had received the AstraZeneca vaccine, the research team concluded that these were extremely rare events. ${ }^{37}$

In another prospective cohort study, comparing the thromboembolism risk of AstraZeneca and Pfizer, it was found that AstraZeneca was found to be linked with small increased risks of Immune Thrombocytopenic Purpura (ITP), arterial thromboembolic and hemorrhagic events. However no 
positive associations were seen between Pfizer and the same events. ${ }^{38}$ A similar study comparing the two vaccines, showed that AstraZeneca was associated with a much higher frequency of adverse reactions compared with that elicited by Pfizer in each group of adverse effects evaluated, including risk of thrombocytopenia, bleeding and thrombosis. Deaths due to these events were concluded to be 0.4 and 4.8 deaths/ 1 million doses, for Pfizer and AstraZeneca recipients, respectively. ${ }^{39}$

According to a study comparing the risk of thrombosis by AstraZeneca, Pfizer and Moderna, the collective rate of cases of thrombotic events per 1 million person vaccinated-days was 0.21 . For venous thromboembolism (VTE) and arterial thromboembolism (ATE), rates were respectively 0.075 and 0.13 cases per 1 million person vaccinated-days (number of thrombotic cases in the given time period divided by the total number of days the vaccinated persons were at risk during the same period). ${ }^{40}$ The study further described that risk of VTE was less than ATE in the two mRNA vaccines while the AstraZeneca vaccine had evenly shared proportions. Moreover, there was an unexpected increase of cerebral venous thrombosis with incidence rates of $1.1 \%, 0.9 \%$, and $0.4 \%$ with AstraZeneca, Moderna and Pfizer vaccines respectively. ${ }^{40}$

A similar study compared the risk of cerebral venous thrombosis (CVT) following SARS CoV-2 diagnosis, influenza infection and receipt of Pfizer or Moderna vaccines. The incidence of portal vein thrombosis (PVT) was also evaluated in these groups, as well as the baseline CVT incidence within a two-week period. It was found that the incidence of CVT following SARS CoV-2 was higher than that observed across the entire records database evaluated. The incidence of CVT after SARS CoV-2 diagnosis was 39.0 per million people and was found to be higher than that after influenza or after receiving Pfizer or Moderna vaccines. For PVT, the incidence was 436.4 per million people after SARS CoV-2, 98.4 after influenza, and 44.9 after Pfizer or Moderna. ${ }^{41}$ All of the above suggest that the risk of thromboembolism by SARS CoV-2 is greater than any increase seen by the vaccines and therefore the benefits far outweigh the risks.

A new entity that has recently been reported is vaccine-induced thrombotic thrombocytopaenia. It is a rare entity mainly reported in individuals who were administered the AstraZeneca vaccine. The patients presented with extensive thrombosis in atypical sites with associated thrombocytopenia and auto-antibodies to platelet-factor 4 (PF4). The mechanism considered responsible for this is the production of PF4 autoantibodies through molecular mimicry and binding of these antibodies to the human FcR $\gamma$ IIA receptor of platelets, which causes platelet aggregation, resulting in thrombosis. ${ }^{42}$ This has been linked to adenoviral vaccines and not been reported with any other vaccine types, but due to the rarity of the entity, generalization without further extensive evaluation is impossible.

Comprehensive reports of thromboembolic risk by Sputnik V and Sinopharm could not be found in the published literature.

With the spread of misinformation on vaccination, subfertility was also considered an adverse effect associated with SARS CoV-2 vaccines. However recent preliminary clinical trials have disproved this notion, with a few published, and multiple pre-publication research indicating that Covid 19 vaccines do not contribute to male or female subfertility. ${ }^{43}$ 


\section{Future perspectives}

Understudied areas include the effect of vaccines on children and pregnant women. However, ethical considerations limit such studies and therefore pose difficulties in providing elaborate safety profiles for any vaccine. A singular study done to assess the adverse effects of the Moderna and Pfizer vaccines on pregnant women revealed that injection site pain was more commonly reported by the pregnant women than non-pregnant women. Other adverse effects were less, and the incidence of adverse pregnancy and neonatal outcomes were not found to be different from those before the pandemic. However, the authors have stressed that more longitudinal studies are needed with larger cohorts and longer follow up periods to effectively come to any conclusion. ${ }^{44}$

\section{Benefits vs. Risks - Indispensability of vaccines}

SARS CoV-2 has provided an ominous future for global health as it threatens to overwhelm health networks of less financially equipped nations. Thus, efficacious vaccination programmes are of extreme importance to avert the imminent health and financial crisis imposed on nations burdened with a large number of new cases. However, vaccines have their own set of limitations and risks including unpredictable outcomes. Multiple large scale multinational cohort studies have confirmed and repeatedly emphasized that Covid 19 vaccines are associated with mortality reduction by SARS CoV-2. When considering the Pfizer and AstraZeneca vaccines, a single dose of either vaccine was $85 \%$ effective at preventing death and $80 \%$ effective in preventing hospital admissions due to severe disease. ${ }^{45}$ Similar findings were reported for Moderna which was $94 \%$ effective in reducing hospital admissions. ${ }^{46}$ Effectiveness of Sputnik V for preventing laboratoryconfirmed infections was $78.6 \%$ while its effectiveness in reducing hospitalizations and deaths was $87.6 \%$ and $84.8 \%$, respectively. ${ }^{47}$ Sinopharm has reported an overall efficacy of $78.1 \% .{ }^{24}$ It is apparent that Covid 19 vaccines are important in reducing morbidity and mortality associated with SARS CoV-2. Considering the adverse reactions discussed above, it is evident that most of these ill-effects are either mild, or rare events. When considering the pros and cons of vaccination it is therefore prudent to summarize that the benefits of vaccination far outweigh the risks, and vaccination remains the preliminary method of prevention of SARS CoV-2 and reducing adverse outcomes of the infection. Improvement of the present vaccines, and creation of new vaccines against SARS CoV-2 is sure to continue, which fosters a belief that the uncertainty of the future may change in favour of optimism, with hope towards a life without the limitations and worries imposed by the SARS CoV-2 pandemic.

\section{Declarations}

Acknowledgements: None

Funding source: None.

Conflict of Interest statement: The authors declare that there are no conflicting interests.

Ethics statement: Ethics approval not required

All authors were involved in the synopsis and writing of the article. Additionally, Prof. S. Dharmaratne was involved in final review. 


\section{References}

1. Harrison AG, Lin T, Wang P. Mechanisms of SARS-CoV-2 transmission and pathogenesis. Trends Immunol. 2020; 41(12):1100-15.doi: https://doi.org/10.1016/j.it.2020.10.004

2. Bennet BM, Wolf J, Laureano R, et al. Review of current vaccine development strategies to prevent coronavirus disease 2019. Toxicol Pathol. 2020; 48(7):800-9.

doi:https://doi.org/10.1177\%2F0192623320959090

3. WHO. WHO coronavirus (COVID-19) dashboard 2021. available from: https://covid19.who.int/

4. $\mathrm{Hu}$ B, Guo H, Zhou P, et al. Characteristics of SARS-CoV-2 and COVID-19. Nat Rev Microbiol. 2021; 19(3):141-54. doi: https://doi.org/10.1038/s41579-020-00459-7

5. Zhou P, Yang X-L, Wang X-G, et al. A pneumonia outbreak associated with a new coronavirus of probable bat origin. Nature. 2020; 579(7798):270-3.

doi: https://doi.org/10.1038/s41586-020-2012-7

6. Hoffmann M, Kleine-Weber H, Schroeder S, et al. SARS-CoV-2 cell entry depends on ACE2 and TMPRSS2 and is blocked by a clinically proven protease inhibitor. Cell. 2020; 181(2):271-280.e8. doi: https://doi.org/10.1016/j.cell.2020.02.052

7. Lukassen S, Chua RL, Trefzer T, et al. SARS-CoV-2 receptor ACE2 and TMPRSS2 are primarily expressed in bronchial transient secretory cells. EMBO J. 2020; 39(10):e105114.

doi: https://doi.org/10.15252/embj.20105114

8. Chen N, Zhou M, Dong X, et al. Epidemiological and clinical characteristics of 99 cases of 2019 novel coronavirus pneumonia in Wuhan, China: a descriptive study. Lancet. 2020; 395(10223):50713. doi: https://doi.org/10.1016/s0140-6736(20)30211-7

9. Yüce M, Filiztekin E, Özkaya KG. Covid-19 diagnosis - a review of current methods. biosens bioelectron. 2021; 172:112752. doi: https://doi.org/10.1016/j.bios.2020.112752

10. To KK-W, Tsang OT-Y, Leung W-S, et al. Temporal profiles of viral load in posterior oropharyngeal saliva samples and serum antibody responses during infection by SARS-CoV-2: an observational cohort study. Lancet Infect Dis. 2020; 20(5):565-74.

doi:https://doi.org/10.1016/s1473-3099(20)30196-1

11. Corman VM, Landt O, Kaiser M, et al. Detection of 2019 novel coronavirus (2019-nCoV) by realtime RT-PCR. Eurosurveillance. 2020; 25(3):2000045.

doi: https://dx.doi.org/10.2807\%2F1560-7917.ES.2020.25.3.2000045

12. Mohamadian M, Chiti H, Shoghli A, et al. Covid-19: Virology, biology and novel laboratory diagnosis. J Gene Med. 2021; 23(2):e3303. doi https://doi.org/10.1002/jgm.3303

13. Jin Y, Yang H, Ji W, et al. Virology, epidemiology, pathogenesis, and control of COVID-19. Viruses. 2020; 12(4):372. doi: https://doi.org/10.3390/v12040372

14. WHO. Draft landscape and tracker of Covid-19 candidate vaccines. R \& D Blueprint. 2021. available from: https://www.who.int/publications/m/item/draft-landscape-of-covid-19-candidatevaccines

15. Anderson EJ, Rouphael NG, Widge AT, et al. Safety and immunogenicity of SARS-CoV-2 mRNA1273 vaccine in older adults. $N$ Engl J Med. 2020; 383(25):2427-38.

doi:https://doi.org/10.1056/NEJMoa2028436

16. Ritchie H, Ortiz-Ospina E, Beltekian D, et al. Coronavirus pandemic: Our World in Data. 2020. Available from: https://ourworldindata.org/coronavirus

17. Polack FP, Thomas SJ, Kitchin N, et al. Safety and efficacy of the BNT162b2 mRNA Covid-19 vaccine. N Engl J Med . 2020; 383(27):2603-15. doi:https://doi.org/10.1056/NEJMoa2034577

18. Jackson LA, Anderson EJ, Rouphael NG, et al. An mRNA vaccine against SARS-CoV-2 -

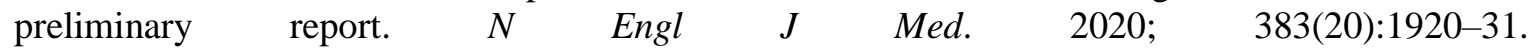
doi:https://doi.org/10.1056/NEJMoa2022483

19. Dai L, Gao GF. Viral targets for vaccines against Covid-19. Nat Rev Immunol. 2021; 21(2):7382.doi: https://doi.org/10.1038/s41577-020-00480-0 
20. Jones I, Roy P. Sputnik V COVID-19 vaccine candidate appears safe and effective. Lancet 2021; 397(10275):642-3. doi: https://doi.org/10.1016/S0140-6736(21)00191-4

21. Logunov DY, Dolzhikova I V, Shcheblyakov D V, et al. Safety and efficacy of an $\operatorname{rad} 26$ and $\operatorname{rad} 5$ vector-based heterologous prime-boost Covid-19 vaccine: an interim analysis of a randomised controlled phase 3 trial in Russia. Lancet. 2021; 397(10275):671-81.

doi:https://doi.org/10.1016/s0140-6736(21)00234-8

22. Voysey M, Clemens SAC, Madhi SA, et al. Safety and efficacy of the ChAdOx1 nCoV-19 vaccine (AZD1222) against SARS-CoV-2: an interim analysis of four randomised controlled trials in Brazil, South Africa, and the UK. Lancet. 2021; 397(10269):99-111.

doi:https://doi.org/10.1016/S0140-6736(20)32661-1

23. Ramasamy MN, Minassian AM, Ewer KJ, et al. Safety and immunogenicity of ChAdOx1 nCoV-19 vaccine administered in a prime-boost regimen in young and old adults (COV002): a single-blind, randomised, controlled, phase 2/3 trial. Lancet. 2020; 396(10267):1979-93.

doi:https://doi.org/10.1016/S0140-6736(20)32466-1

24. Xia S, Zhang Y, Wang Y, et al. safety and immunogenicity of an inactivated SARS-CoV-2 vaccine, BBIBP-CorV: a randomised, double-blind, placebo-controlled, phase 1/2 trial. Lancet Infect Dis. 2021; 21(1):39-51. doi: https://doi.org/10.1016/S1473-3099(20)30831-8

25. Sahin U, Muik A, Derhovanessian E, et al. Covid-19 vaccine BNT162b1 elicits human antibody and TH1 T cell responses. Nature. 2020; 586(7830):594-9.

doi: https://doi.org/10.1038/s41586-020-2814-7

26. Sui Y, Bekele Y, Berzofsky JA. Potential SARS-CoV-2 immune correlates of protection in infection and vaccine immunization. Pathog. 2021; 10(2):138.

doi: https://doi.org/10.3390/pathogens10020138

27. Vályi-Nagy I, Matula Z, Gönczi M, et al. Comparison of antibody and T cell responses elicited by BBIBP-CorV (Sinopharm) and BNT162b2 (Pfizer-BioNTech) vaccines against SARS-CoV-2 in healthy adult humans. GeroScience. 2021; 43(5):2321-2331. doi: https://doi.org/10.1007/s11357021-00471-6

28. Deng Y, Li Y, Yang R, et al. SARS-CoV-2-specific T cell immunity to structural proteins in inactivated COVID-19 vaccine recipients. Cell Mol Immunol. 2021; 18(8):2040-1.

doi:https://doi.org/10.1038/s41423-021-00730-8

29. Woldemeskel BA, Garliss CC, Blankson JN. SARS-CoV-2 mRNA vaccines induce broad CD4+ T cell responses that recognize SARS-CoV-2 variants and HCoV-NL63.

J Clin Invest. 2021; 131(10):e149335. doi: https://doi.org/10.1172/jci149335

30. Yan Z-P, Yang M, Lai C-L. Covid-19 vaccines: a review of the safety and efficacy of current clinical trials. Pharmaceuticals 2021; 14(5):406. doi: https://doi.org/10.3390/ph14050406

31. Krammer F. SARS-CoV-2 vaccines in development. Nature. 2020; 586(7830):516-27. doi:https://doi.org/10.1038/s41586-020-2798-3

32. Nilsson L, Csuth Á, Storsaeter J, et al. Vaccine allergy: evidence to consider for Covid-19 vaccines. Curr Opin Allergy Clin Immunol. 2021; 21(4):401-9.

doi:https://doi.org/10.1097/aci.0000000000000762

33. Yacoub M-R, Cucca V, Asperti C, et al. Efficacy of a rational algorithm to assess allergy risk in patients receiving the BNT162b2 vaccine. Vaccine. 2021; 39(44):6464-9.

doi:https://doi.org/10.1016/j.vaccine.2021.09.048

34. Shavit R, Maoz-Segal R, Iancovici-Kidon M, et al. Prevalence of allergic reactions after PfizerBioNTech COVID-19 vaccination among adults with high allergy risk. JAMA Netw Open. 2021; 4(8):e2122255-e2122255. doi:https://doi.org/10.1001/jamanetworkopen.2021.22255

35. Klimek L, Novak N, Cabanillas B, et al. Allergenic components of the mRNA-1273 vaccine for COVID-19: possible involvement of polyethylene glycol and IgG-mediated complement activation. Allergy. 2021; doi: https://doi.org/10.1111/all.14794

36. Pottegård A, Lund LC, Karlstad $\varnothing$, et al. Arterial events, venous thromboembolism, 
thrombocytopenia, and bleeding after vaccination with Oxford-AstraZeneca ChAdOx1-S in Denmark and Norway: Population based cohort study. BMJ. 2021; 373:1-10.

doi:https://doi.org/10.1136/bmj.n1114

37. Tobaiqy M, Elkout H, Maclure K. Analysis of thrombotic adverse reactions of COVID-19 AstraZeneca vaccine reported to EudraVigilance database. Vaccines. 2021; 9(4):1-8.

doi: https://doi.org/10.3390/vaccines 9040393

38. Simpson CR, Shi T, Vasileiou E, et al. First-dose ChAdOx1 and BNT162b2 Covid-19 vaccines and thrombocytopenic, thromboembolic and hemorrhagic events in Scotland. Nat Med . 2021; 27:12901297 doi:https://doi.org/10.1038/s41591-021-01408-4

39. Cari L, Fiore P, Naghavi Alhosseini M, et al. Blood clots and bleeding events following BNT162b2 and ChAdOx1 nCoV-19 vaccine: an analysis of European data. $J$ Autoimmun. 2021; 122:102685. doi:https://doi.org/10.1016/j.jaut.2021.102685

40. Smadja DM, Yue Q-Y, Chocron R, et al. Vaccination against Covid-19: insight from arterial and venous thrombosis occurrence using data from VigiBase. Eur Respir J. 2021; 2100956.

doi:https://doi.org/10.1183/13993003.00956-2021

41. Taquet M, Husain M, Geddes JR, et al. Cerebral venous thrombosis and portal vein thrombosis: a retrospective cohort study of 537,913 Covid-19 cases. medRxiv. 2021; 2021.04.27.21256153.

doi: https://doi.org/10.1101/2021.04.27.21256153

42. Dotan A, Shoenfeld Y. Perspectives on vaccine induced thrombotic thrombocytopenia. J Autoimmun. 2021; 121:102663. doi: https://doi.org/10.1016/j.jaut.2021.102663

43. Lifshitz D, Haas J, Lebovitz O, et al. Does mRNA SARS-CoV-2 vaccine detrimentally affect male fertility, as reflected by semen analysis. Reprod Biomed Online. 2021;

doi:https://doi.org/10.1016/j.rbmo.2021.09.021

44. Shimabukuro TT, Kim SY, Myers TR, et al. Preliminary findings of mRNA Covid-19 vaccine safety in pregnant persons. $N$ Engl J Med. 2021; 384:2273-2282 doi:https://doi.org/10.1056/NEJMoa2104983

45. Lopez Bernal J, Andrews N, Gower C, et al. Effectiveness of the Pfizer-BioNTech and OxfordAstraZeneca vaccines on Covid-19 related symptoms, hospital admissions, and mortality in older adults in England: test negative case-control study. BMJ. 2021; 373:n1088.

doi:https://doi.org/10.1136/bmj.n1088

46. Tenforde MW, Olson SM, Self WH, et al. Effectiveness of Pfizer-BioNTech and Moderna vaccines against Covid-19 among hospitalized adults aged $\geq 65$ years-united states, 2021. MMWR Recomm Reports. 2021; 70(18):674-9. doi:https://doi.org/10.15585/mmwr.mm7018e1

47. González S, Olszevicki S, Salazar M, et al. Effectiveness of the first component of Gam-COVIDVac (Sputnik V) on reduction of SARS-CoV-2 confirmed infections, hospitalisations and mortality in patients aged 60-79: a retrospective cohort study in Argentina. EClinicalMedicine. 2021; 40:101126. doi: https://doi.org/10.1016/j.eclinm.2021.101126 